\title{
Road Intersections as Pervasive Computing Environments: towards a Multiagent Real-Time Collision Warning System
}

\author{
Flora Dilys Salim, Licheng Cai, Maria Indrawan \\ Faculty of Information Technology \\ Monash University \\ Caulfield East, VIC, Australia
}

\author{
Seng Wai Loke \\ Computer Science and Computer Engineering \\ La Trobe University \\ Bundoora, VIC, Australia
}

\begin{abstract}
Embedded with sensors and appropriate computational entities, a road intersection can be viewed as a pervasive computing environment. The crash rate in road intersections demonstrates the need for a fast and accurate collision detection system. We suggest that an intersection collision detection system should be able to adapt to different types of intersections for faster collision detection. Moreover, a real-time application-level communication protocol to warn affected drivers is required. An intersection agent that takes vehicular status information from vehicle agents and learns, detects and warns collisions at a road intersection is proposed. The issues, challenges, and cost of a multiagent collision avoidance system are discussed. A communication protocol that is designed specifically with intersection safety in mind is presented here.
\end{abstract}

Multiagent system; collision warning and avoidance; communication protocol; cost model; road intersection safety

\section{INTERSECTIONS AT STAKE}

The rate of fatalities of road intersection collisions has not significantly changed in more than two decades, regardless of improved intersection design, innovation of vehicles, and more sophisticated Intelligent Transportation System (ITS) technology. Intersections are among the most hazardous sites on U.S. roads. The statistic of crashes in the year 2002 in the USA reported that 50 percents of all reported crashes, approximately 3.2 million crashes, were intersection-related. 22 percents of the total fatalities on the road, which was 9,612 fatalities, and roughly 1.5 million injuries and 3 million collisions, happened at intersection surroundings ${ }^{1}$. The high accident and fatality rate in intersections is chiefly determined by the complexity at each intersection. Therefore, the complex nature of intersection collisions requires systems that warn drivers about possible collisions. In addition, given the uniqueness of each intersection, rather than manually finetuning a system for each intersection, an intelligent system for intersection safety should be able to adapt to different types of intersections automatically [1].

U.S. Department of Transportation - Federal Highway Administration, Institute of Transportation Engineers, Intersection Safety Briefing Sheet, April 2004, [Online],

http://safety.fhwa.dot.gov/intersections/interbriefing/index.htm
There have been a number of initiatives in developing intersection collision warning systems and/or avoidance systems. Currently, no existing intersection collision warning and avoidance systems can tackle intersection collision problems entirely. Intersection collision warning and avoidance systems can be categorized as either vehicle-based, infrastructure-only or as infrastructure-vehicle cooperative [1]. Many existing intersection collision warning systems [2, 3] are still infrastructure-only systems, and are limited in many factors, mainly due to inexistence of communication between road infrastructure and vehicles and inability of the systems to adapt to different types of intersections. Vehicle-based intersection collision warning systems are fairly effective for a single vehicle as there's no any communication means between vehicle and infrastructure. However, in an intersection, the potential danger normally impacts more than one vehicle; therefore, a cooperative system is preferred [1]. To our knowledge, existing research projects in cooperative systems for intersection safety do not mention techniques to discover crash patterns and pre-crash behaviour associations, which are essential to detecting and reacting to potential threats [1]. Existing intersection collision warning and avoidance systems are still deficient in these areas: incomprehensive data source, element of learning and adaptability, performance and scalability, real-time communication protocol, and relationship between detection and warning. Consequently, we need to have a cooperative intersection collision warning and avoidance system that: incorporates data from vehicle and roadside sensors, is generic and adaptable to various intersections, is able to perform real-time detection and warning no matter how busy an intersection is, has an established real-time communication protocol, is able to send a warning message effectively and efficiently in order to avoid a future immediate collision.

We have proposed the U\&I Aware Framework [4], as pictured in Figure 1. The U\&I Aware Framework consist of three main components: learning, detection, and warning of collisions in an intersection. The main difference of our framework with existing collision warning and avoidance systems is the learning component that enables the system to be generic and adaptive to different types of intersections and assists faster collision detection process. In the early papers we 
concentrate our discussion on the learning [5] and detection [6] component of the system. In this paper, we introduce communication and cost model of delivering warning to vehicles in intersection once a potential collision is detected the U\&I Aware Framework.

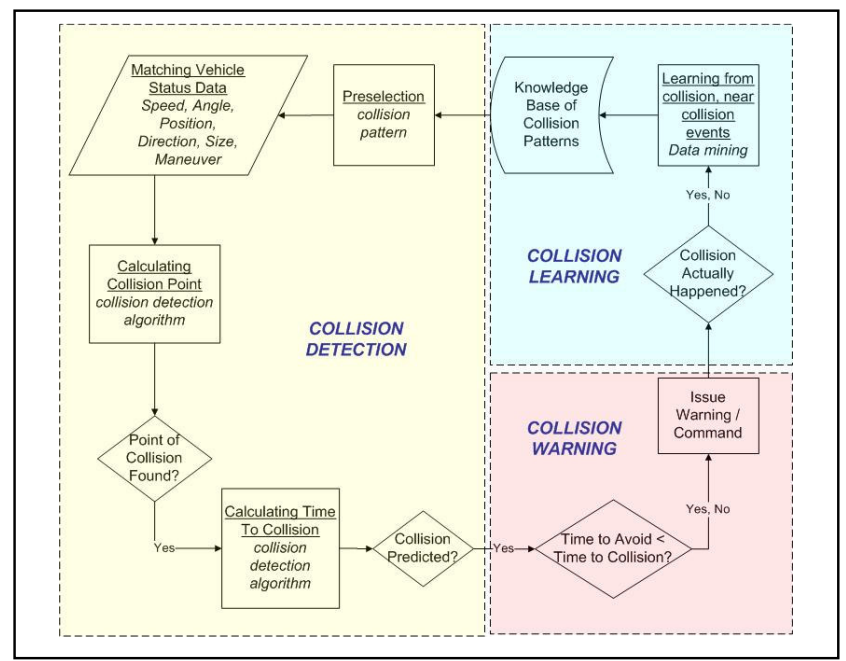

Figure 1. Learning, Detection, and Warning in U\&I Aware Framework

\section{Challenges In Delivering REAL-Time WARNING}

As the time needed to avoid collision (TTA) should be less than the elapsed time to the collision (TTC) in order to avoid a collision, we need to increase the speed of detection (thus increasing the TTC value) and reduce the communication cost (thus lessening TTA value). In order to achieve a generic and real-time framework for intersection safety, it is apparent that:

- to speed up collision detection (increasing TTC), we need: centralised location of computations, reduction of number of vehicle pairs for collision detection by preselection method, and knowledge base and learning of collision patterns [6]

- $\quad$ to reduce the time needed to avoid collision (reducing TTA), we need: accurate cost models of Time-ToAvoid (TTA) to achieve timely warning or command message and real-time communication protocol. This is the purpose of the discussion in this section.

Collision detection misses should be avoided; however the number of alarms should also be regulated, as too many false alarms can cause a nuisance to the driver [7]. If collision is detected, it is important to realise whether the time and distance needed to respond to the warning and avoid the collision is sufficient [2]. Time-To-Collision (TTC) is computed by the pair-wise collision detection algorithm [2]. Time-ToAvoidance (TTA) in Miller and Huang's peer-to-peer collision warning system [2] is computed based on vehicle kinetics, network latency, and human response time. If TTC is much greater than TTA, warning is not issued. However, if TTC is close to TTA and driver is not braking, then warning is issued. Otherwise, if TTC is less than TTA, mitigation unit is executed to lessen the collision effect [2]. The best timing to warn drivers vary based on driver's skills and experience, therefore they also proposed a parameter $\gamma$ for tweaking the timing of effective warning, as in (1) [2].

$$
\text { TTC- TTA }<\gamma
$$

When $\gamma$ is large the algorithm will be more conservative. When it is too conservative, it can be a distraction rather than assistance to a driver. Thus $\gamma$ must be adjusted well based on the best probable driver experience. The formula to calculate TTA proposed by Miller and Huang [2] is:

$$
T T A=t_{r}+\frac{\beta v}{\mu g}
$$

where $t_{r}$ is the response time of the driver, $\beta$ is the speed reduction factor (its range is from 0 to 1 that indicates the level of brake), $v$ is the current speed, $\mu$ is the anticipated tire-road friction coefficient, $g$ is the acceleration of gravity. However, there are different factors that can be considered in calculating TTA as proposed by INTERSAFE [3]. The minimum warning distance required to inform a driver in order to stop in front of the intersection or behind the stop line [3], is:

$$
D_{\text {inf ormation }}=\frac{V_{0}^{2}}{2 a}+\left(t_{\text {driver }}+t_{\text {machine }}+t_{\text {inf ormation }}\right) \times V_{0}
$$

where $v_{0}$ is the velocity of the vehicle, $a$ is the vehicle braking deceleration, $t_{\text {driver }}$ is the driver's response time to brake, $t_{\text {machine }}$ is the combination of braking system and warning system response time, and $t_{\text {information }}$ is the constant information time, which is a time determined by the assistance system to allow the driver to react and prepare the driver to stop. Similarly, the formula (3) can be translated to calculate TTA by summing $t_{\text {driver }}, t_{\text {machine }}$, and $t_{\text {information }}$ with the current vehicle speed divided by deceleration rate.

The $t_{r}$ factor in Miller and Huang's formula (2) is the same as $t_{\text {driver }}$ in INTERSAFE's formula (3). The main difference between INTERSAFE formula and Miller and Huang's proposal are that Miller and Huang do not consider $t_{\text {information, }}$, which is necessary in a warning system, and secondly, $t_{\text {machine }}$ in INTERSAFE is more comprehensive by including warning system response time. The time components in INTERSAFE's TTA formula have been evaluated on real-world tests and can be used as a point of reference in our system. Nevertheless, none of both algorithms consider communication or messaging cost with external systems such as traffic control or central component. In Miller and Huang's, it is because their system is vehicle-based warning system. However, in INTERSAFE, some sorts of central intersection computer system is assumed, therefore communication cost should also be included in the cost model of TTA. As TTA is greatly determined by velocity and distance to the collision point, sometimes, rather than issuing a warning to the drivers of the affected vehicles, it can be more useful to issue a command to the relevant vehicles to so that appropriate action can be taken automatically rather 
than wait for the user to react to a warning message (thereby adding a further delay). For example, if TTC is greater than TTA, warning message will be generated for driver to take an action. However, if the TTC is less than TTA, then the system can issue a command to activate the braking system. The higher is the velocity; the lower is the available time to avoid collision, and a greater chance that a direct command message to the machine can be more effective. Therefore, we take into account these issues into our cost model of collision avoidance, which is discussed in the next section.

\section{Cost MOdel of COLlision AvOIDANCE}

When a collision is detected, it is important that we only send messages to affected vehicles. For collision warning particularly, point-to-point messaging is used between vehicle and intersection agents instead of broadcasting. As there is a need for real-time warning, the messages sent are short and thus, only requires a short processing time. However, when TTA is not enough to issue a warning to notify the driver, it is better to send a command message to the vehicle agent directly to brake. Therefore we propose two types of avoidance messages with two types of TTA accordingly (Fig. 2), which are: (1) Warning message, intended for driver, measured by

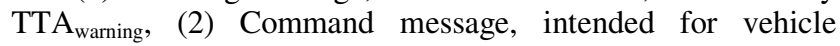
braking system, measured by $\mathrm{TTA}_{\text {command. }}$ So, when should a warning or command message be generated, the rule of thumb to follow is as follows:

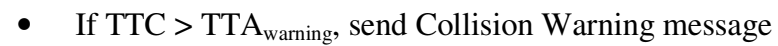

- If TTC $<=\mathrm{TTA}_{\text {warning, }}$, send Command message

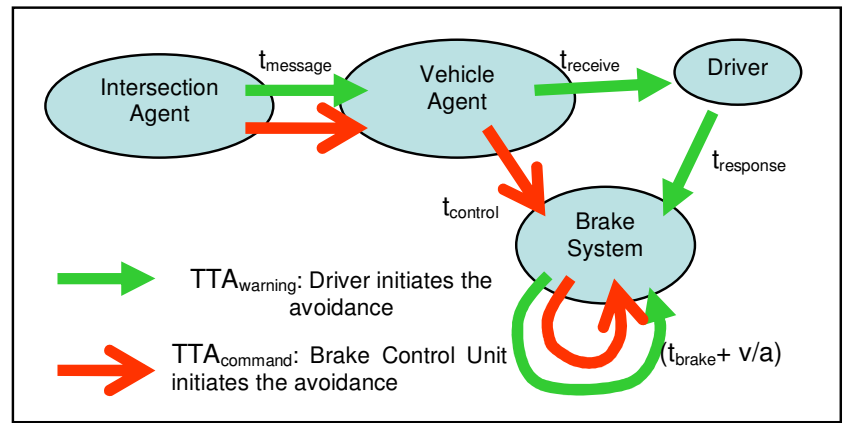

Figure 2. TTA Cost Model Diagram

We propose the cost model for $\mathrm{TTA}_{\text {warning }}(4)$, where driver initiates the avoidance, which is as follows:

$$
T T A_{\text {warning }}=t_{\text {message }}+t_{\text {receive }}+t_{\text {response }}+t_{\text {brake }}+\frac{v}{a}
$$

where $t_{\text {message }}$ is the required time to generate, transmit and read a warning message by the software, $t_{\text {receive }}$ is the time for a driver to receive the message, $t_{\text {response }}$ is the response time for a driver to take an action, $t_{\text {brake }}$ is the response time of braking system, and $v / a$ is the time to full stop ( $v$ is velocity and $a$ is acceleration). The cost model for $\mathrm{TTA}_{\text {command }}(5)$, where the Brake Control Unit initiates the avoidance, is:

$$
T T A_{\text {command }}=t_{\text {message }}+t_{\text {control }}+t_{\text {brake }}+\frac{v}{a}
$$

where $t_{\text {control }}$ is the response time of brake control unit. The cost of issuing a warning (in time units) after notification of new information or event $\left(t_{\text {message }}\right)$ is computed by (6):

$$
t_{\text {message }}=t_{\text {generate }}+t_{\text {transmit }}+t_{\text {read }}
$$

where $t_{\text {generate }}$ is time to generate the message, $t_{\text {transmit }}$ is time for message transmission, and $t_{\text {read }}$ is time for the vehicle's computer to read the message. $t_{\text {transmit }}$ can be calculated by (7):

$$
t_{\text {transmit }}=\frac{\text { message } \_ \text {size }}{\text { bandwidth }}
$$

where message_size is the size of the message in bits, and bandwith is the capacity of the communication channel in bits per second. The next section discusses the structure of the messages transmitted in U\&I Aware Framework.

\section{Message Protocol}

When considering communication between the components in the intersection collision warning system, which include vehicle components and the central component, we must consider all the possible components involved and the model of the communication. We implement agent based communication model in this protocol. It is necessary to know where to host the computations for collision detection, not only to reduce computation cost of collision detection but also to reduce the cost of generating warnings. In order to have a global bird's eye view of the intersection and reduce the overhead of communicating vehicle's sensor data to each other (which is the case if distributed approach is adopted), it is recommended to apply centralized computation. Thus, in order to have a centralized location of computations, U\&I Aware Framework uses a central component that is located in the intersection vicinity. This central component is called the intersection agent in our proposed framework. Agent is a piece of software that can act autonomously on behalf of the user. Similarly, an intersection agent represents traffic authority to detect threats and warn possible affected vehicles of incoming hazards. The vehicle agent of each car in the intersection should always report to the intersection agent of its entry into and exit from a designated area in the vicinity of the intersection and also send its status periodically. Vehicle information and driver's behavior information, such as driving maneuvers are retrieved from in-vehicle sensors. An intersection agent manages the tasks of communication, learning, detection, and warning. The communication between the intersection agent and vehicle agents are regulated inside the administration zone (Fig. 3) which is the spatial domain that determines the region of authority of an intersection agent to coordinate vehicle agents in the approaching and passing vehicles.

As the intersection agent needs to works together with all the vehicle agents in vicinity, there are at least three different messaging required between the vehicle agents and intersection agent. First, status messages need to be sent periodically from vehicles to central component to keep the central component up to date of vehicle's data for collision detection calculation. Since the status message is sent periodically, we need to also 
consider if the message is initiated by vehicle agents (push method) or the intersection agent (pull method). In real-time terms, it is better to employ push method, as the request message from the intersection agent is eliminated, thus reducing communication cost. The second type of the message that is required is the registration message. The presence of each vehicle needs to be known to the intersection agent. Third, warning messages from the intersection agent sent to vehicle components. At this point, there are two options of message delivery: point-to-point or broadcast. As false warning or alarms need to be avoided as much as possible, broadcast is not an option for collision warning. Point-to-point message delivery can ensure that only affected vehicles will be warned. However, if there is general warning (e.g. weather warning or speed limit warning) needs to be issued then broadcast can be used. We also need to consider the message protocol to use, as it is important to consider the effectiveness and efficiency of the message. We need to create a lightweight message protocol for intersection collision warnings. At this stage, there has no real-time messaging protocol that has been designed specifically for intersection collision warning. We propose three types of messages (Fig. 4) transmitted within the administration zone, which are: status report, registration, and warning report.

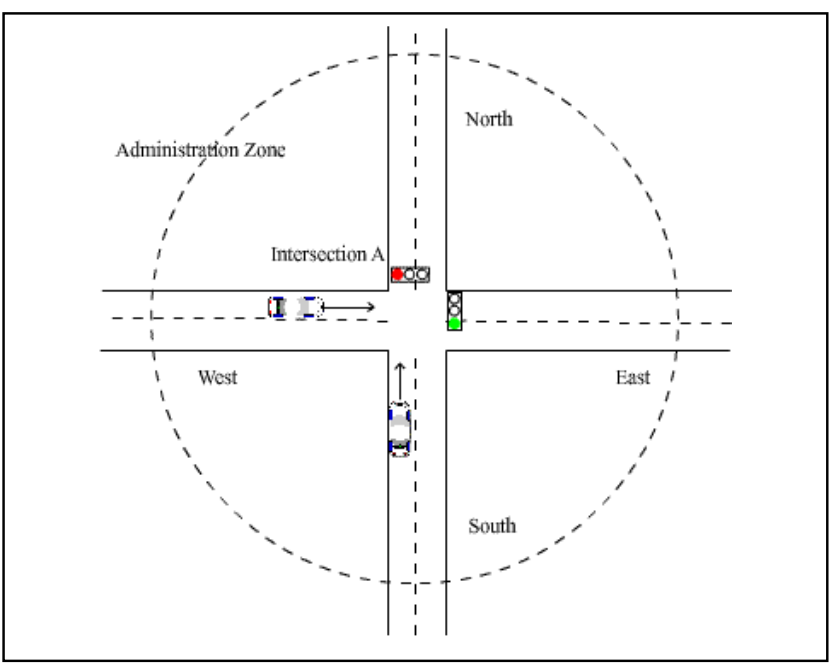

Figure 3. Intersection Agent's Administration Zone

\section{A. Status Message}

When a Vehicle Agent (VA) receives an Intersection Agent (IA)'s signal, it sends a status message to the IA to report its status every specified interval time, e.g. 6 milliseconds. This message includes the vehicle's dynamic information: vehicle ID, speed, position, angle, and maneuver. When all the required information can be retrieved from roadside sensors, status report from the vehicle agent is no longer necessary, as the intersection agent is getting the data straight from the roadside sensors. The message structure is:

status $\mid<$ vehicle ID $>|<x>|<y>\mid<$ speed $>\mid<$ acceleration $>$ $\mid<$ direction $>\mid<$ angle $>\mid<$ maneuver $>$
The word "status" is to indicate the message type. The vehicle $I D$ is the registration number of the vehicle, e.g. "VICABC001". The $x$, y values are the coordinate values of the vehicle's position in our simulated environment, e.g. 213, 320. The speed is the velocity of the vehicle, e.g. 16.666. Its unit is meter/second. The acceleration is the acceleration of the vehicle, e.g. 1.471. Its unit is meter/second ${ }^{2}$. The direction is the travel direction of the vehicle, e.g. 0.00 (towards north). If the vehicle travels towards east, the direction value is 90.00 ; towards south, 180.00, towards west, 270.00. The angle is the steering angle of the vehicle, e.g. 0.00 for going straight. If the vehicle turns 5 degree to the left, the value is -5.00 . If it turns 5 degree to the right, the value is 5.00. The maneuver is the intended driving maneuver that is predicted by in-vehicle devices 1 second before it occurs [8]. The value of maneuver includes Passing, TurnLeft, TurnRight, ChangeLaneLeft, ChangeLaneRight, Starting, and Stopping. Each parameter is separated by a vertical bar "l".

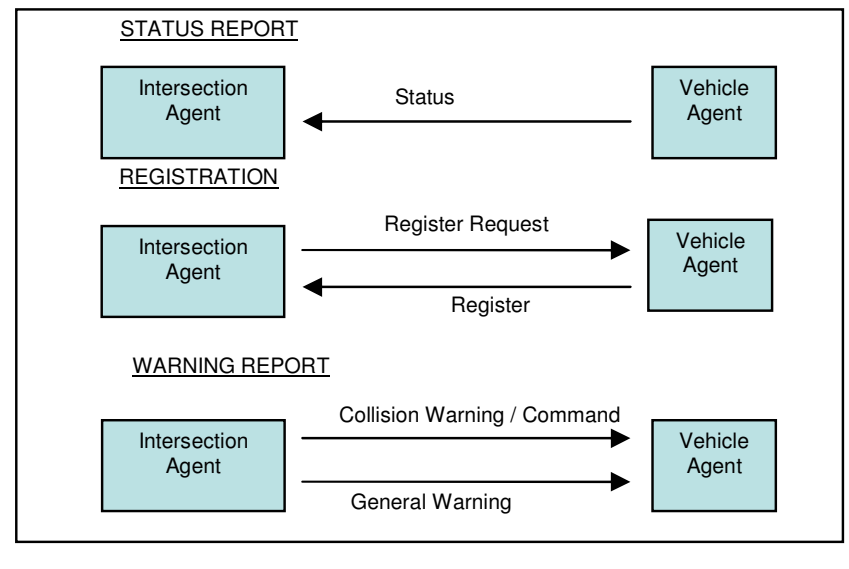

Figure 4. Message Protocol

\section{B. Registration message}

After the IA receives a VA status message, the IA checks its own database for the static information of the vehicle, such as the size of the vehicle. If it does not have this information, it sends a Registration Request message to the vehicle. When a vehicle receives a Registration Request message from an IA, it replies it with a Registration message. The message includes the vehicle's static information: vehicle ID, size.

The content of the Register Request Message is very simple. Its structure is:

regreq $\mid<$ vehicle $I D>$

The word "regreq" is to indicate the message type.

The Register Message includes the vehicle's static information, such as vehicle ID and size. The message structure is:

\section{regist $\mid<$ vehicle ID> | <length $>\mid<$ width $>$}

The word "regist" indicates the message type. The length is the length of the vehicle in meter. The width is the width of the vehicle in meter. 


\section{Warning report}

There are two types of warning reports. Firstly, General Warning message, which is broadcast to all vehicles including information for speed limit and drunk driver warning. The message structure can be one type of followings:

- spdlmt | <value>, e.g. "spdlmt|60.000" means that speed limit is 60 kilometers per hour

- $\quad d r k d r v \mid<$ vehicle $I D>\mid<x$ value $>\mid<y$ value $>$, e.g. "drkdrv IVICPAD123|2211578" means that a drunk driver is driving vehicle "PAD-123" at the position $(221,578)$

Secondly, Collision Avoidance message, which can either be a Collision Warning or Command message. If an intersection system detects that a collision will happen, its IA send Collision Warning to notify the driver of the pair of involved vehicles. This message includes data: vehicle ID, Time-To-Collision (TTC), collision position, and collision type. The message structure is:

\section{collwn $\mid<$ vehicle ID $>|<T T C>|<x>|<y>|<$ type $>$}

The word "collwn" is used to indicate the message type. The TTC is the time to collision for the particular vehicle. The $x, y$ are the position of the collision point in our simulated environment. The type is the collision type, e.g. Side or RearEnd. The vehicle agent receives it, processes it, and warns the driver (Fig. 5).

However, if the TTA is less than the TTC, the IA sends a Command message to the VA so that the vehicle takes an action automatically without the driver's intervention. This message includes data: vehicle ID and action. The message structure is:

\section{commnd | <vehicle ID > | <acceleration >}

The word "commnd" indicates the message type. If acceleration is negative, the vehicle needs to slow down. Otherwise, the vehicle needs to speed up. All the protocols have been implemented on the simulated intersection agent and vehicle agent for the evaluation of the communication cost.

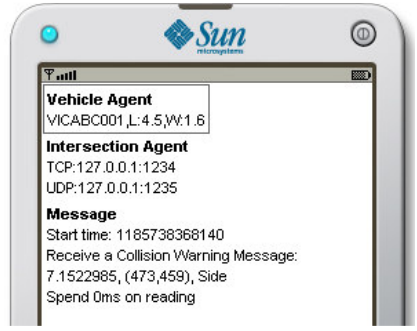

Figure 5. Vehicle Agent Collision Warning Test

\section{EVAluAtion}

In the proposed TTA cost model in Section III, there are several components contributing to the $\mathrm{TTA}_{\text {warning }}$ and TTA $_{\text {command. }}$ Only one of these components $-t_{\text {message }}$ such as in formula (4), (5), (6) is affected by our proposed communication protocol. The values of other components are constant and can be obtained from the INTERSAFE project [3]. In our prototype, the $t_{\text {generate }}$ and $t_{\text {read }}$ are measured. Both are less than 1 millisecond. The $t_{\text {transmit }}$ can be calculated using the formula (7). The size of the Collision Warning message can be calculated based on its structure which is described in the Section IV.C. This message consists of 40 characters approximately. Since we use UTF-8 for encoding, each character can be encoded by using one byte. Therefore, the size of the message is 40 bytes approximately. If the bandwidth is $10 \mathrm{Mbit}$ per second, the $t_{\text {transmit }}$ will be 0.032 milliseconds. If consider the extra wrapper size from the TCP / IPv6 and other lower layer protocol, the $t_{\text {transmit }}$ is around 0.11 milliseconds. The $t_{\text {message }}$ is so small comparing to the other components that it is negligible. This exhibits the efficiency of our real-time communication protocol.

According to the INTERSAFE project [3], based on realworld experimentations, the value range for $t_{\text {response }}, t_{\text {brake }}$ and $1 / a$ (gravity acceleration) are as displayed in the Table I [3]. $t_{\text {receive }}$ value is 1.1 seconds, since that is the average reaction time for elderly [9]. Most of other drivers will have smaller value of $t_{\text {receive }}$ than 1.1 seconds. Hence, if the current speed is $60 \mathrm{~km} / \mathrm{h}(16.67 \mathrm{~m} / \mathrm{s})$, the minimum value of $T T A_{\text {warning }}$ is 4.630 seconds and the maximum value of $T T A_{\text {warning }}$ is 9.084 seconds when our proposed communication model and protocol are employed. Most intersections have speed limit $40 \mathrm{~km} / \mathrm{h}$. In order to reduce TTA greatly, velocity must be reduced, as our communication protocol yields negligible cost. Nevertheless, if the TTAwarning is larger than or equal to TTC, there is no enough time to inform the driver to avoid the collision. Therefore, a command message is going to be sent directly to the vehicle agent, and then the Brake Control Unit in the vehicle initiates the brake action to stop the vehicle directly without driver's interruption. Given the current speed of 60 $\mathrm{km} / \mathrm{hr}$, the minimum value of TTAcommand is 2.73 seconds and the maximum value of TTAcommand is 5.984 seconds.

TABLE I. TTA COMPONENTS VALUE RANGE (ADAPTED FROM [3])

\begin{tabular}{|l|l|c|}
\hline & \multicolumn{1}{|c|}{ Min. Value } & Max. Value \\
\hline$t_{\text {receive }}$ & \multicolumn{2}{|c|}{1.1 seconds (average reaction time for elderly) } \\
\hline$t_{\text {response }}$ & 0.8 second & 2 second \\
\hline$t_{\text {brake }}$ & 0.3 second & 0.5 second \\
\hline$a$ & $0.31 \mathrm{~g}=3.038 \mathrm{~m} / \mathrm{s}^{2}$ & $0.7 \mathrm{~g}=6.86 \mathrm{~m} / \mathrm{s}^{2}$ \\
\hline $1 / a$ & $1 /(6.86 \mathrm{~m} / \mathrm{s} 2)=$ & $1 /\left(3.038 \mathrm{~m} / \mathrm{s}^{2}\right)=$ \\
& $0.1458 \mathrm{~s} 2 / \mathrm{m}$ & $0.329 \mathrm{~s} 2 / \mathrm{m}$ \\
\hline
\end{tabular}

We measure TTC in our simulated prototype. Whenever a future collision event is detected for the first time, it is recorded in a $\log$ file. Then, the average of detection time (time to collision) for each run is calculated. In each execution, the average time to collision is calculated. If preselection is ignored in collision detection, the average time to collision is 5.6 seconds. However, when preselection is used, the average time to collision is 8.7 second, which is around 3 seconds earlier than the previous method. Therefore, preselection is proven to speed up the process of collision detection. The 
greater the number of vehicles in an intersection, the more preselection is useful and effective. When TTC is greater than TTA, collision can be avoided. The average figure of TTC shows that collision can be avoided most of the time when our proposed real-time communication protocol and preselection algorithm are applied.

\section{CONCLUSION}

We have proposed the Ubiquitous Intersection Awareness (U\&I Aware) framework, which is a generic and real-time context-aware framework for collision detection and warning at road intersections. In order to have real-time collision avoidance, Time-To-Avoidance (TTA) should be less than Time-To-Collision (TTC), therefore we need to increase TTC by quickening the collision detection process (so available time before collision is greater) and decrease TTA by shortening communication and warning process. A real-time communication protocol has been proposed with the cost model of TTA accordingly. As a result, impending collisions can be avoided in real world situations.

\section{REFERENCES}

[1] F. D. Salim, S. Krishnaswamy, S. W. Loke, A. Rakotonirainy, "ContextAware Ubiquitous Data Mining Based Agent Model for Intersection Safety”, Proc. of EUC 2005 Workshops, LNCS, Springer, 2005.
[2] R. Miller and Q. Huang, "An Adaptive Peer-to-Peer Collision Warning System", Proc. of Vehicular Technology Conference (VTC) Spring 2002, Birmingham, Alabama.

[3] INTERSAFE, D40.4 Requirements for intersection safety applications, 28 Oct 2005.

[4] F. D. Salim, S. W. Loke, A. Rakotonirainy, S. Krishnaswamy, "U\&I Aware: A Framework Using Data Mining and Collision Detection to Increase Awareness for Intersection Users", Proc. of the 21st International Conference on Advanced Information Networking and Applications Workshops (AINAW'07), 2007, pp. 530-535.

[5] F. D. Salim, S. W. Loke, A. Rakotonirainy, S. Krishnaswamy, "Simulated Intersection Environment and Learning of Collision and Traffic Data in the U \& I Aware Framework" , Proc. of The 4th International Conference on Ubiquitous Intelligence and Computing (UIC-07), Hong Kong, July 2007.

[6] F. D. Salim, S. W. Loke, A. Rakotonirainy, B. Srinivasan, S Krishnaswamy, "Collision Pattern Modeling and Real-Time Collision Detection at Road Intersections", Proc. of The 10th International IEEE Conference on Intelligent Transportation Systems, Seattle, Washington, USA, IEEE Computer Society Press, 2007.

[7] R. v. d Horst and J. Hogema, "Time-To-Collision and Collision Avoidance Systems", Proc. of the 6th ICTCT (International Cooperation on Theories and Concepts in Traffic Safety) Workshop, Salzburg, Austria, 1993.

[8] N. Oliver and A. Pentland, "Graphical Models for Driver Behavior Recognition in a SmartCar", Proc. of IEEE Intl. Conference on Intelligent Vehicles 2000, Detroit, Michigan, October 2000.

[9] M. Green, "How Long Does It Take To Stop? Methodological Analysis of Driver Perception-Brake Times", Transportation Human Factors, 2000, pp.195-216. Also available at http://www.visualexpert.com/Resources/reactiontime.html 